\title{
Dual Action Additives for Jet A-1: Fuel Dehydrating Icing Inhibitors
}

Sonia L. Repetto, Rishi Patel, Tim Johnson, James F. Costello, Joseph K.-W. Lam, and Christopher J.

Chuck

\section{SUPPORTING INFORMATION}

Table S1. $\quad$ FDII presented in ascending order of catalytic coefficient $k_{\mathrm{H}+}$, along with the corresponding hydrolysis products (i.e., potential icing inhibitors). Calculated log $K_{\mathrm{ow}}$ and standard deviation presented alongside the structures. Experimentally determined values reported in the literature in parentheses [33].

\begin{tabular}{|c|c|c|c|c|}
\hline & FDA & $\log K_{\text {ow }}$ & $\begin{array}{l}\text { Corresponding hydrolysis } \\
\text { products }\end{array}$ & $\log K_{\text {ow }}$ \\
\hline 1 & & $1.88 \pm 0.52$ & $\underbrace{\mathrm{OH}}_{\mathrm{P} 3}$ Butanone & $\begin{array}{l}0.34 \pm 0.11 \\
(0.29) \\
-0.89 \pm 0.25 \\
(-1.04)\end{array}$ \\
\hline 22 & & $\begin{array}{l}0.11 \pm 0.19 \\
(0.25)\end{array}$ & & $\begin{array}{l}-0.12 \pm 0.08 \\
(0.03)\end{array}$ \\
\hline 3 & & $\begin{array}{l}1.24 \pm 0.30 \\
(1.20)\end{array}$ & $\mathrm{H}^{\prime}$ & $\begin{array}{l}0.31 \pm 0.02 \\
(0.23)\end{array}$ \\
\hline
\end{tabular}




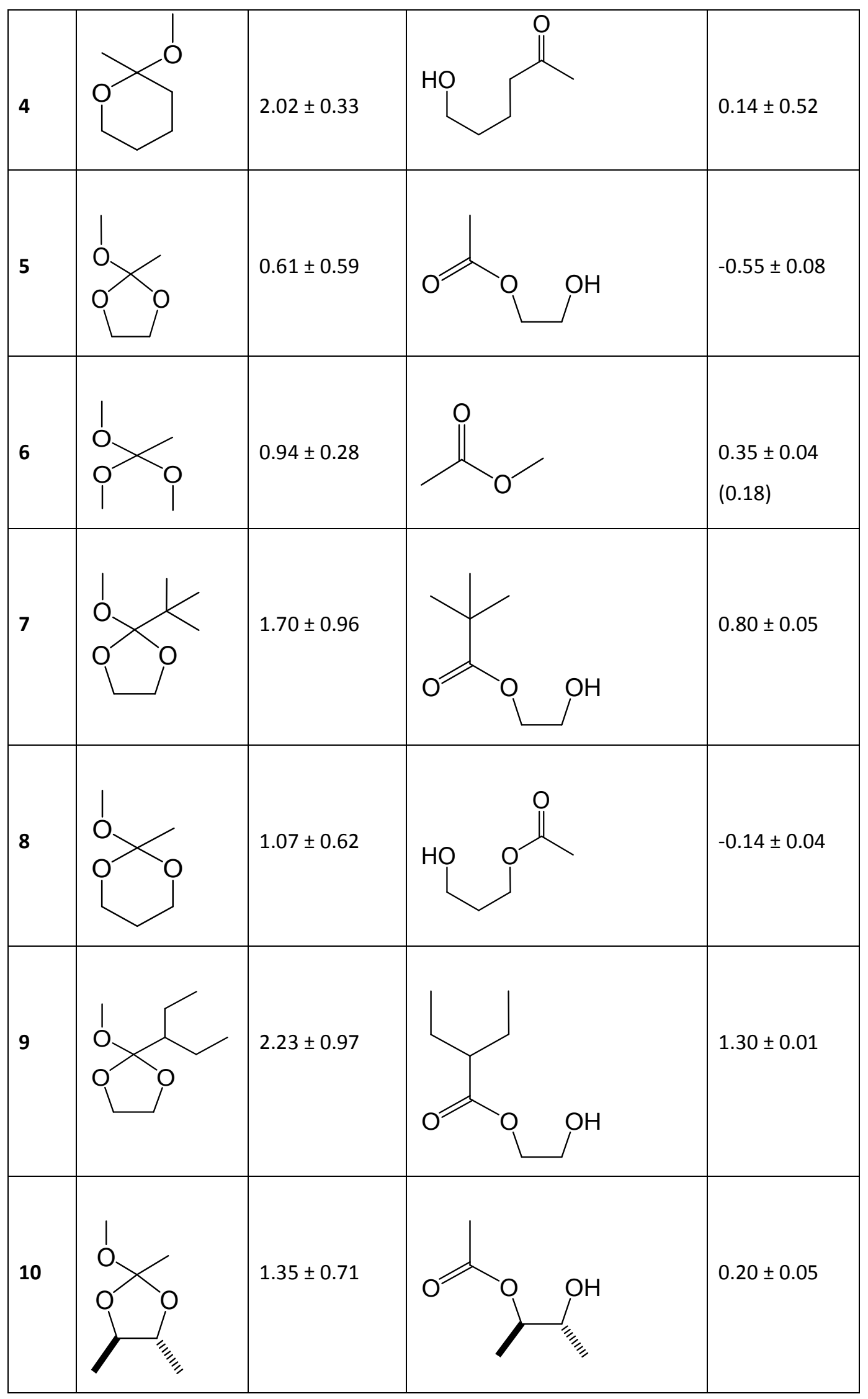




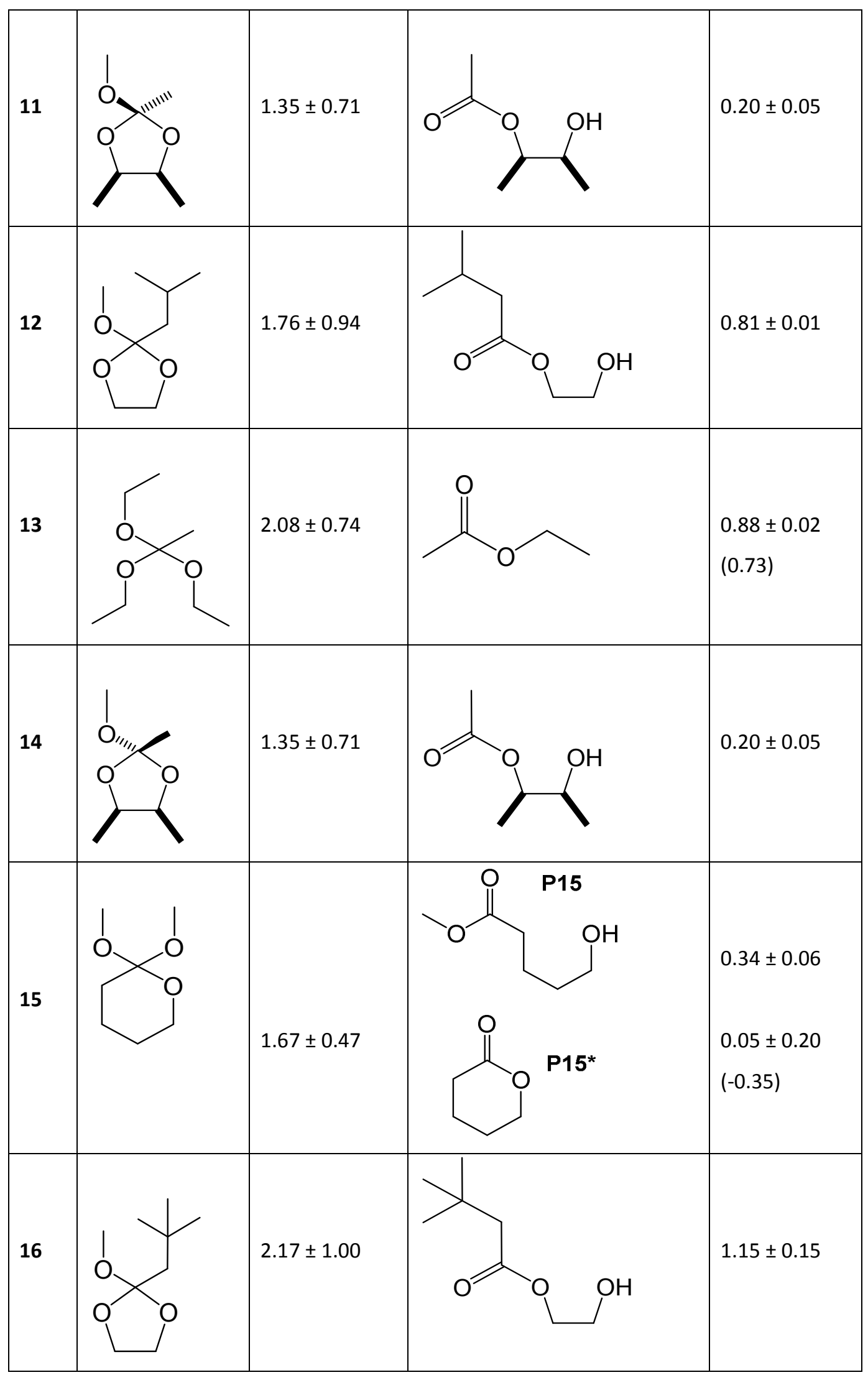


Table S2. Equilibrium data for fuel surrogate/water/FDII hydrolysis products, added water content is in addition to $59 \mathrm{ppm}$ present in the jet fuel.

\begin{tabular}{|c|c|c|c|c|c|}
\hline Compound & $\begin{array}{c}\text { Temperature } \\
\left({ }^{\circ} \mathrm{C}\right)\end{array}$ & $\begin{array}{l}\text { Added } \\
\text { water } \\
\text { content } \\
(\mathrm{v} / \mathrm{v})\end{array}$ & $\begin{array}{c}\text { Initial conc } \\
(\mathrm{mmol} / \mathrm{l})\end{array}$ & $\begin{array}{c}\text { Conc } \\
(\mathrm{mmol} / \mathrm{l}) \text { in } \\
\text { fuel }\end{array}$ & $\begin{array}{c}\text { Conc } \\
(\mathrm{mmol} / \mathrm{l}) \text { in } \\
\mathrm{H}_{2} \mathrm{O}\end{array}$ \\
\hline $\mathrm{MeOH}$ & 5 & 90 & 47.7 & 42.7 & 55828 \\
\hline $\mathrm{EtOH}$ & 5 & 90 & 34.3 & 32.9 & 14722 \\
\hline AP5 & 5 & 90 & 23.2 & 20.3 & 32085 \\
\hline AP8 & 5 & 90 & 24.5 & 24.0 & 5623 \\
\hline $\mathrm{MeOH}$ & 5 & 260 & 47.7 & 34.7 & 49936 \\
\hline $\mathrm{EtOH}$ & 5 & 260 & 34.3 & 28.7 & 21220 \\
\hline AP5 & 5 & 260 & 23.2 & 15.8 & 28108 \\
\hline AP8 & 5 & 260 & 24.5 & 19.6 & 18778 \\
\hline $\mathrm{MeOH}$ & 5 & 500 & 47.7 & 26.5 & 42366 \\
\hline $\mathrm{EtOH}$ & 5 & 500 & 34.3 & 24.6 & 19241 \\
\hline AP5 & 5 & 500 & 23.2 & 11.9 & 22549 \\
\hline AP8 & 5 & 500 & 24.5 & 15.9 & 17202 \\
\hline $\mathrm{MeOH}$ & 25 & 90 & 44.7 & 44.6 & 732 \\
\hline $\mathrm{EtOH}$ & 25 & 90 & 36.0 & 35.8 & 2505 \\
\hline AP5 & 25 & 90 & 42.5 & 41.5 & 10698 \\
\hline AP8 & 25 & 90 & 40.9 & 40.4 & 6398 \\
\hline $\mathrm{MeOH}$ & 25 & 260 & 44.7 & 40.1 & 17474 \\
\hline EtOH & 25 & 260 & 36.0 & 34.6 & 5325 \\
\hline AP5 & 25 & 260 & 42.5 & 37.8 & 17949 \\
\hline AP8 & 25 & 260 & 40.9 & 37.2 & 14461 \\
\hline $\mathrm{MeOH}$ & 25 & 500 & 44.7 & 31.6 & 26178 \\
\hline $\mathrm{EtOH}$ & 25 & 500 & 36.0 & 30.4 & 11164 \\
\hline AP5 & 25 & 500 & 42.5 & 28.7 & 27434 \\
\hline AP8 & 25 & 500 & 40.9 & 33.0 & 15908 \\
\hline
\end{tabular}

